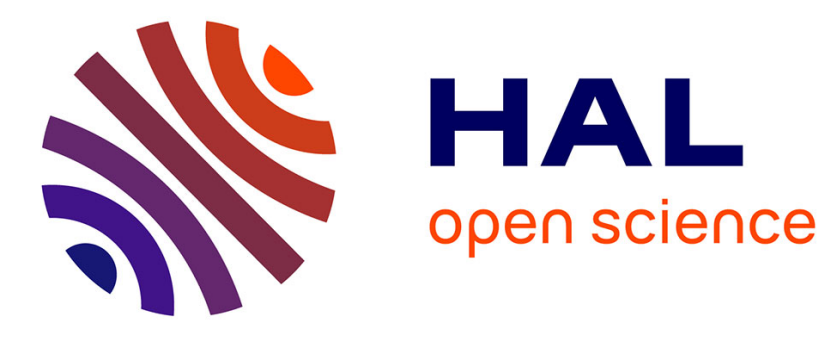

\title{
Les Alpes, de la société de la vache au parc animalier
}

Isabelle Mauz

\section{To cite this version:}

Isabelle Mauz. Les Alpes, de la société de la vache au parc animalier. Raphaël Larrère, Bernadette Lizet, Martine Berlan-Darqué. Histoire des parcs nationaux. Comment prendre soin de la nature, Quae, pp.187-204, 2009. halshs-00382943

\section{HAL Id: halshs-00382943 https://shs.hal.science/halshs-00382943}

Submitted on 11 May 2009

HAL is a multi-disciplinary open access archive for the deposit and dissemination of scientific research documents, whether they are published or not. The documents may come from teaching and research institutions in France or abroad, or from public or private research centers.
L'archive ouverte pluridisciplinaire HAL, est destinée au dépôt et à la diffusion de documents scientifiques de niveau recherche, publiés ou non, émanant des établissements d'enseignement et de recherche français ou étrangers, des laboratoires publics ou privés. 


\title{
Les Alpes, de la société de la vache au parc animalier
}

\author{
Isabelle Mauz \\ Cemagref - UR DTM \\ 2, rue de la Papeterie \\ BP 76 \\ 38402 Saint-Martin-d'Hères cedex \\ isabelle.mauz@grenoble.cemagref.fr
}

Les Alpes deviendront-elles le parc animalier de l'Europe ? La question peut surprendre, à l'heure où l'érosion de la biodiversité est unanimement constatée et déplorée. Mais la grande faune sauvage jouit, dans les Alpes, d’une prospérité inédite et inégalée en Europe occidentale. Mais les touristes sont nombreux, qui ne demandent qu’à voir et à approcher des animaux sauvages en liberté. Mais des techniques ont été mises au point, ou sont en passe de l'être, qui permettent de gérer et de contrôler efficacement et discrètement les animaux sauvages. Ces éléments, développés tour à tour dans la première partie du texte, donnent à penser que la question mérite au moins d’être posée. Entendons-nous bien. Je n’imagine pas que les Alpes seront un jour encloses. Si je parle de parc animalier, c'est qu'il n'existe pas (encore ?), à ma connaissance, de terme parfaitement adapté à ce qui émerge actuellement. Dès 1992, Anne Vourc’h et Valentin Pelosse avançaient l'expression de «zoos du troisième type » pour désigner l'apparition d'un nouveau dispositif de contrôle et de mise en scène des animaux sauvages (1992 : 129) : à côté des cages des ménageries, telles celles du Jardin des plantes (zoos du premier type), et des enclos élargis des parcs de vision, tel celui de Peaugres (zoos du deuxième type), prennent désormais place des parcs animaliers sans clôture et étendus à l'espace rural tout entier (zoos du troisième type). La formule a le mérite de la force mais la situation, dans les Alpes, rappelle peut-être en effet davantage celle des parcs animaliers, qui ont fleuri un peu partout au cours des dernières décennies, et qui se distinguent des zoos par leur étendue et par la semi (simili)-liberté que l’on y accorde aux animaux.

L’évolution récente de la grande faune sauvage ne va pas sans susciter des réactions fortement contrastées au sein des sociétés alpines : ceux qui l’ont encouragée se félicitent; certains s’y adaptent progressivement et tentent d'en tirer parti tandis que d'autres s'inquiètent, et tentent de résister à l'avancée des animaux sauvages et aux façons de dire et de faire qui l’accompagnent. Je présenterai ces grands types de réactions dans la seconde partie du texte. Je m’interrogerai, pour finir, sur la possibilité de concilier cette nouvelle définition des Alpes, avec celle, pluriséculaire, de région dévolue à l’élevage des troupeaux domestiques (Abry et al., 1998).

Mais il me faut, avant tout, souligner la nécessité d’être prudent. De manière générale, on le sait, l'environnement est l'un des domaines où règne une grande incertitude (Callon et al., 2001 ; Fischer, 2000). Quelle sera la répartition des animaux sauvages dans une ou deux générations ? Quels seront leur comportement et celui des hommes à leur égard ? Il est extrêmement difficile de le prédire. Il suffit, pour s’en convaincre, de se 
tourner vers un passé qui n’est pas si lointain. Dans les années soixante, personne n'imaginait que la grande faune sauvage se multiplierait, s'étendrait et se diversifierait comme elle l'a fait. On ne voyait guère alors en montagne que des chamois, des renards, des blaireaux et, pour les régions les mieux pourvues, quelques rares bouquetins. Il y a seulement vingt ans, ce qui fait une très courte génération humaine, on prenait l'arrivée des loups dans les Alpes pour de la science-fiction. Dans une thèse vétérinaire soutenue en 1984, et consacrée au prédateur, j’ai relevé la mention suivante: «imaginons une France soudainement repeuplée en loups. En admettant que ces derniers ne soient pas exterminés sur le champ, ce qui est actuellement fort improbable, ils seraient très rapidement contaminés par des renards enragés » (Loire, 1984 : 94). Officiellement, les loups sont arrivés dans les Alpes françaises en novembre 1992. Bien des indices donnent cependant à penser qu'ils étaient là, au moins épisodiquement, depuis plusieurs années ; en 1984, ils n’étaient probablement plus très éloignés de la frontière franco-italienne. C’est dire si les personnes les mieux informées peuvent se tromper.

\section{Les éléments d'un zoo idéal}

Il n'en reste pas moins que la grande faune sauvage ne s'était pas aussi bien portée dans les Alpes depuis fort longtemps. Le tableau d'ensemble que l'on peut brosser en quelques lignes demanderait certes à être nuancé, selon les espèces et selon les lieux, la densité des animaux pouvant décroître, voire chuter, lorsqu'on s'éloigne des aires protégées et de leur immédiate périphérie ${ }^{1}$. Mais des espèces qui se trouvaient déjà dans les Alpes, notamment le chamois et le bouquetin, sont aujourd'hui nettement mieux représentées que par le passé. D’autres, exterminées à la fin du dix-neuvième siècle et au début du siècle dernier, se réinstallent progressivement, spontanément ou à la faveur de réintroductions.

\section{Une situation inédite de la grande faune sauvage}

On rencontre désormais plusieurs espèces d'ongulés encore récemment inconnues des montagnards, comme les cerfs, les chevreuils et les sangliers, ainsi que de grands rapaces, comme les aigles royaux, les vautours fauves et les gypaètes barbus et, last but not least, de grands carnivores, comme les lynx et les loups. Notons, à ce propos, que les ours slovènes montreraient des tendances migratrices - je ne parle pas des quelques animaux introduits dans les Pyrénées mais de ceux qui, par leurs propres moyens, se dirigent vers l'Italie et l'Autriche voisines (Jerena et al., 2003). Enfin, des espèces ont découvert les Alpes, comme le mouflon, que des chasseurs, des protecteurs et des forestiers ont introduit dans plusieurs massifs à partir des années 1950 (Massol, 2005), ou semblent sur le point de le faire. Si l'on accepte de légèrement s'écarter des Alpes pour gagner le Jura français, on peut signaler qu'un chien viverrin (Nyctereutes procyonides) y a été trouvé mort en août 2003. Les chiens viverrins sont originaires d'Extrême Orient et ils ont été acclimatés en Russie occidentale dans la première moitié du vingtième siècle (Lecomte, 1989), comme animal à fourrure (Hainard, 1997 : 225) et destructeur de rongeurs (de Planhol, 2004 : 313). Cas isolé et destiné à le rester ou prodrome de nouvelles colonisations animales ? Il est trop tôt pour le dire. Quoi qu'il en soit, les animaux circulent décidément beaucoup et les Alpes, si peuplées et fréquentées soient-elles en ce début de troisième millénaire, leur plaisent visiblement.

Les grands animaux ne sont pas seulement plus variés et plus nombreux. En bien des endroits, ils se sont acclimatés à la présence humaine et ils se laissent approcher plus facilement qu’auparavant, ne craignant plus des

1 C’est, par exemple, le cas du bouquetin (Girard, $2000: 11$ ). 
hommes devenus à leur égard majoritairement débonnaires. En zone centrale des parcs nationaux, les marmottes, jadis reléguées dans les pentes et dans les alpages, s’installent dans les prés de fauche encore exploités par des agriculteurs. Celles qui régulièrement voisinent avec les randonneurs n’hésitent pas à quémander des friandises voire, pour les plus hardies, à fouiner elles-mêmes dans les sacs. Au printemps, les bouquetins descendent dans les vallées y profiter de l'herbe tôt verdie et s'attardent dans les luzernières, sans être apparemment gênés par la proximité des habitations. Quant aux renards, ils furètent la nuit dans les stations de sports d'hiver et dans les villages, se régalant des poubelles bien garnies. Bref, les marques d'une certaine familiarisation et d'une certaine “commensalisation ” (de Planhol, 2004 : 260) des animaux sauvages se multiplient.

Cette évolution tant quantitative que qualitative de la grande faune sauvage n'est pas propre aux Alpes et concerne en réalité toute l'Europe occidentale, avec bien sûr des variations de degré et des contre-exemples². Elle résulte de la conjonction d'une série de facteurs - la création d'espaces protégés, la protection et la réintroduction d'espèces, l'adoption de plans de chasse, l'abandon des secteurs les plus défavorables à l'agriculture intensive et leur reconquête par la friche ou par la forêt - qui ne s'exercent pas tous nécessairement partout et qui se combinent différemment selon les lieux et les espèces. Il se trouve que la plupart de ces facteurs sont, dans les Alpes, à la fois particulièrement anciens et singulièrement marqués. Ainsi, trois cents espaces protégés couvrent approximativement $15 \%$ de l'arc alpin ${ }^{3}$ et les premiers d'entre eux, le parc national suisse de l'Engadine et le parc national italien du Grand Paradis, datent de près d'un siècle. En France, le doyen des parcs nationaux, celui de la Vanoise, a fêté en 2003 son quarantième anniversaire. Les introductions, les réintroductions et les renforcements ont été abondants et précoces. Quantité de bouquetins ont été lâchés dans de nombreux massifs, dès le début du vingtième siècle en Suisse, à partir des années soixante en France. Des castors, des cerfs, et des chevreuils ont, entre autres, été réintroduits un peu partout. Ces opérations, qui ont connu au fil du temps un succès grandissant, se poursuivent aujourd'hui, avec des espèces déjà bien implantées, comme le bouquetin et le cerf, et avec des espèces encore rares et dont la réintroduction n’est à peu près maîtrisée que depuis peu, comme le gypaète barbu. Plusieurs espèces protégées ont en outre une aire de répartition essentiellement alpine, pour l'instant du moins. C'est notamment le cas du bouquetin, du gypaète barbu et du loup. Enfin, l'activité agricole a beaucoup régressé dans les Alpes, maintes surfaces anciennement cultivées n'étant plus aujourd'hui ni fauchées, ni pâturées par les troupeaux domestiques. Seuls les plans de chasse ont été adoptés plus tardivement qu'ailleurs mais ils ont alors souvent entraîné une augmentation significative des populations.

L'essor de la grande faune sauvage s'explique ainsi conjointement par la volonté de la protéger et de la restaurer et par la réduction, sur de vastes surfaces, de la pression de culture et d’élevage. Il n’a pas été tellement freiné par l'urbanisation et l'équipement des Alpes, beaucoup d'animaux s'étant révélés capables de traverser les milieux les plus anthropisés, voire de s'y installer, à partir du moment où ils n'en étaient pas chassés. Contrairement à une idée communément répandue, l'urbanisation et l'ensauvagement ne sont pas antagonistes mais tendent plutôt, jusqu'à un certain point, à se renforcer mutuellement. Situées « au cœur de l'Europe », les

2 Des évolutions voisines sont rapportées ailleurs dans le monde, avec des conséquences économiques, sociales, et politiques, elles aussi similaires : des animaux ayant acquis un statut d'espèce protégée s'enhardissent et se multiplient; un tourisme animalier se développe, qui profite à certains habitants mais qui provoque le ressentiment d'une grande partie des agriculteurs et des éleveurs. Pour des exemples africains, voir (Luxereau, 2004), (Kleitz, 2003), (Madden, 2004).

3 Source : réseau alpin des espaces protégés, www.alparc.org 
Alpes apparaissent en définitive comme une région particulièrement propice au développement des grands animaux sauvages, et certains n’hésitent pas à parler de leur nouvelle « vocation » : « Mais beaucoup plus encore que les forêts, où l'exploitation du bois introduit des contraintes, l'espace qui s'offre de plus en plus aujourd'hui à la grande faune sauvage européenne est celui de l'étage altitudinal élevé. Largement déserté désormais par les hommes, si l'on excepte les foyers très ponctuels de stations sportives d'activité saisonnière, et de quelques centres industriels de vallées, presque totalement abandonné par les cultures et voué au mieux à une exploitation pastorale qui se maintient difficilement, il procure à l'animal, en dehors même des Parcs, et pour peu qu'une protection satisfaisante lui soit assurée contre des excès éventuels de la chasse, la tranquillité et la paix. Au cœur de l’Europe, les Alpes, les Carpathes, les Pyrénées et les chaînes Cantabriques, voire les massifs anciens de la zone hercynienne (du Massif Central aux Vosges et à ceux de l'Allemagne moyenne), constitueront désormais un immense domaine voué de plus en plus largement à la vie sauvage. Il en sera de même des montagnes axiales de la Péninsule scandinave. C’est là que se trouvent l’immense majorité des Parcs Nationaux européens » (de Planhol, 2004 : 645).

\section{Un public nombreux, avide de voir des animaux sauvages en liberté}

En 1871 paraissait à Londres le livre de Leslie Stephen, Le terrain de jeu de l'Europe. Devenues au cours de la seconde moitié du vingtième siècle, le terrain de jeu, non plus seulement d'une élite intellectuelle et sportive, mais de foules de touristes venus de toute l'Europe et, pour les sites les plus prestigieux, du monde entier, les Alpes n’ont jamais autant mérité le titre que leur a donné l'écrivain et alpiniste anglais (Debarbieux, 1995 : 43).

D’emblée, les Alpes et les ongulés sauvages qui n’existaient pas ailleurs ont été étroitement associés. L'observation de ces animaux, même fugitive, même de très loin, apparaît dans les comptes rendus de courses effectuées par des adhérents du Club alpin français comme un événement digne d'être relaté. Les alpinistes ont d'ailleurs été parmi les tout premiers à réclamer la création d'espaces protégés pour ménager les chamois et sauver les bouquetins, éteints ou en voie d'extinction dans la quasi totalité de l'arc alpin au début du vingtième siècle (Mauz, 2003 : 33-34). L'idée que l'on ne peut aller dans les Alpes sans y rencontrer des espèces singulières a en outre été largement diffusée par la littérature, puis renforcée par les responsables des espaces protégés, qui systématiquement les mettent en scène dans la présentation des territoires dont ils ont la charge. Les touristes ont parfaitement assimilé qu'ils doivent voir des animaux sauvages dans les Alpes, en particulier lorsqu'ils vont dans les espaces protégés et il n'est pas étonnant qu'une partie d'entre eux déclarent aujourd'hui s’y rendre dans cette intention, et qu'ils s’y intéressent avant tout à la grande faune.

Les grands animaux sauvages des Alpes ont d'autant plus de succès que les zoos sont sévèrement critiqués et contestés (Baratay et Hardouin-Fugier, 1998). La progression de la sensibilité écologique et la montée des préoccupations pour le bien-être animal se sont accompagnées d'une répugnance croissante du public à voir des animaux captifs et qui ont été manifestement déplacés. La fascination pour les animaux sauvages n’a en rien diminué mais on les veut désormais dans leur milieu et avec toutes les apparences de la liberté et de la naturalité. Les animaux doivent être faciles à approcher, sans être séparés du spectateur par des grillages ni des fossés. C'est pourquoi les Alpes, avec leurs animaux de plus en plus nombreux, de plus en plus familiers, et libres, constituent une manière de zoo idéal, dont cherchent désespérément à se rapprocher les gestionnaires des 
établissements classiques ${ }^{4}$. Là, les visiteurs peuvent satisfaire leur désir d'animaux sauvages sans éprouver la culpabilité de les voir prisonniers.

\section{Une “ éco-zoo-technie ” de plus en plus performante}

Il n’y a pas seulement, dans les Alpes, d'un côté des animaux sauvages nombreux et variés, de l'autre des spectateurs venus en masse pour les regarder. Des connaissances ont été acquises et des techniques de repérage, de suivi et de gestion des animaux ont été mises au point, instaurant une nouvelle relation à l'animal sauvage, qui n'est ni de la chasse, ni de l'élevage ni de la protection stricte et qui pourtant leur ressemble à certains égards : l'animal sauvage est souvent nommé et sa reproduction peut être régulée (cf. infra), comme dans l'élevage ; il peut être capturé et manipulé, comme dans la chasse mais il n'est pas tué, si ce n’est par accident et une grande attention est accordée à sa survie et à sa tranquillité. Disons, faute de mieux, qu’il est « géré ». Cette gestion de l'animal sauvage ne repose pas sur un corps homogène de savoirs et de savoir-faire, du moins pour l'heure, mais plutôt sur une nébuleuse, qu'André Micoud (1993) a proposé de désigner par le terme d' « éco-zoo-technie ». On appellera donc « éco-zoo-techniciens » les initiateurs, les expérimentateurs et de manière générale les artisans de cette éco-zoo-technie montante, qui peuvent être des scientifiques, des vétérinaires et des gestionnaires de la faune sauvage, ou des chasseurs.

Les animaux sauvages sont de plus en plus observés. Les longues-vues se sont répandues ; les agents des parcs nationaux en sont tous équipés et ils en font régulièrement profiter les visiteurs, qui peuvent ainsi regarder des scènes de la vie animale dans d'excellentes conditions. Des caméras thermiques, permettant d'enregistrer le comportement nocturne des animaux, commencent elles aussi à être utilisées ${ }^{5}$. Des animaux (marmottes, lagopèdes, chamois, bouquetins, etc.) sont par ailleurs munis de dispositifs permettant de les suivre individuellement, de reconstituer leurs déplacements, de préciser leur organisation sociale. D’abord frustes et très voyantes, les techniques de marquage se sont peu à peu perfectionnées. Après avoir apposé sur les oreilles des bouquetins des étiquettes colorées, on les équipe aujourd'hui de colliers radio-émetteurs ou de colliers GPS ${ }^{6}$, ces derniers recueillant des données automatiquement et aussi souvent qu’on le souhaite.

On assiste parallèlement à une miniaturisation et à une distanciation de ces dispositifs. Les marmottes ont d’abord été marquées avec de la peinture ; elles ont ensuite été équipées de colliers et d'étiquettes auriculaires ; un chercheur explique que des transpondeurs sont à présent insérés sous leur peau, afin d'éviter les pertes et de garantir une meilleure durabilité du suivi : "c'est une petite puce en fait, un peu comme les codes-barres qu'on passe aux caisses des magasins, donc j'ai un lecteur, que je passe sur le transpondeur. Je lis le numéro de la puce et je sais quelle marmotte c'est, parce que souvent elles s'arrachent, en fait, les barrettes métalliques, donc c'est un double-système si vous voulez, en général. Donc ça a une durée de vie illimitée, puisque c'est inerte et c'est activé par le lecteur. » Désormais tout petit, le matériel identificateur peut être fixé à l'intérieur du corps de l'animal et être ainsi totalement invisible. Mieux encore : on parvient à identifier et à suivre des animaux

4 La rénovation du zoo de Vincennes, actuellement partiellement fermé en raison de sa vétusté, est ainsi régie par la volonté de préserver le bien-être animal, « d'évoquer le mieux possible le milieu naturel » et « d'immerger le visiteur dans l'écosystème qui lui est présenté », Le Monde, 26-27 juin 2005.

5 Des interactions entre des chiens de protection et des loups ont ainsi pu être observées, de nuit, dans le parc national du Mercantour. Ces observations ont donné lieu à un film, intitulé : Entre chiens et loups. Film du parc national du Mercantour réalisé par Sylvain Menoud.

6 Global positioning system. 
sauvages, sans capture ni matériel incorporé d’aucune sorte, grâce aux méthodes d’analyse génétique « non invasives $»^{7}$ mises au point pour l’ours des Pyrénées, puis pour le loup. Il est probable que de telles méthodes, aujourd'hui encore relativement lentes et coûteuses, seront à l'avenir plus rapides, plus accessibles et qu'elles se banaliseront. Elles ne sont pas sans exercer des effets sur la perception des animaux. Le public ignore souvent qu'une partie d'entre eux ont été réintroduits et qu'ils sont, pour certains, équipés d’un matériel de suivi. Or, les étiquettes auriculaires et les colliers émetteurs des bouquetins et des marmottes peuvent gêner le spectateur, parce qu'ils rappellent trop fortement que les animaux sont manipulés et qu'ils ne sont pas si sauvages qu'on le voudrait. Les fils que nous attachons aux animaux sauvages rendus imperceptibles, l'illusion de la naturalité sera parfaite. Les animaux seront suivis, contrôlés (Bobbé, 2004 : 207-208), mais discrètement, à l’insu du spectateur.

Les éco-zoo-techniciens ne se contentent pas de repérer les animaux sauvages, de les observer et de les suivre. Il leur arrive de les gérer. Jusqu’à tout récemment, ils se sont essentiellement employés à favoriser des animaux sauvages rares ou menacés et c'est dans ce domaine qu'ils ont accompli les plus grands progrès. Au cours du vingtième siècle, ils ont acquis tout un savoir et tout un savoir-faire autour de la reconstitution ${ }^{8}$ d'espèces sauvages menacées de disparition. Ils ont appris à soigner des rapaces malades ou blessés, à capturer, à transporter et à réintroduire différentes espèces, ayant chacune leurs exigences et leurs préférences. Pour le bouquetin, les apprentis réintroducteurs ont d’abord essayé d'élever des animaux en captivité, avant d'opter pour des captures en milieu naturel, par lacets ou par cages-trappes puis par téléanesthésie. Pour le gypaète barbu, le cheminement s’est opéré en sens inverse : les tentatives de capture et de translocation s’étant toutes soldées par des échecs, c'est à partir d’oiseaux détenus par les différents zoos européens que l'on a réussi à produire, en volière, des jeunes qui ont ensuite été relâchés en Autriche, en Italie, en France et en Suisse. Mais il a d’abord fallu trouver comment déterminer le sexe des adultes, comment les apparier et comment marquer les jeunes dont on voulait suivre le devenir.

S’il y a eu des déboires ${ }^{9}$, notamment dans les années qui ont suivi les premières tentatives pour une espèce donnée, les résultats ont souvent fini par dépasser les espérances initiales, si bien que les éco-zoo-techniciens se trouvent confrontés, ici ou là, à l’abondance de certaines espèces hier encore absentes, menacées ou peu communes. Il arrive qu'une intervention s’avère alors nécessaire, qui ne vise plus à renforcer une population, mais au contraire à la réduire ou à la limiter, sans pour autant la menacer. Il s'agit là d’un second type

7 L’identité d’un animal peut être déterminée à partir des traces d'ADN contenu dans les excréments ou dans les poils qu’il a déposés sur le terrain. Aucun prélèvement n’étant effectué sur le corps lui-même, on parle de méthode non invasive.

8 Reconstitués, les animaux sauvages le sont parfois littéralement. Chez une espèce comme la vache, il ne subsiste pas d'animaux sauvages que l'on pourrait réintroduire: l'animal n'existe plus que sous sa forme domestique. Certains ont entrepris de combler ce vide en recourant à des races existantes très rustiques, comme ces vaches écossaises du marais Vernier, présentées par leurs introducteurs comme de grands herbivores sauvages tout droit sortis de la préhistoire et particulièrement aptes à maintenir le marais dans un état jugé favorable (Lizet, 1997). D’autres sont allés plus loin, en prétendant reconstituer l'ancêtre sauvage irrémédiablement disparu, l'aurochs (Bos primigenius), à partir de races censées en être restées les plus proches (Daskiewicz et Aikhenbaum, 1998). Dans son livre sur les mammifères sauvages d'Europe, le naturaliste et artiste animalier Robert Hainard consacre à l'aurochs tout un chapitre, qui se clôt sur la phrase suivante : « Mais la disparition de l'aurochs sauvage étant accomplie, cette œuvre de reconstitution me semble magnifique et pleine de sens » (Hainard, 1997 : 412).

9 L'échec d'une tentative de reconstitution d'une population peut avoir de multiples causes, par exemple l'incapacité d'animaux ayant vécu en captivité de s'adapter à leurs nouvelles conditions de vie (bouquetins), le départ ou le décès d'animaux que l’on a déplacés (bouquetins, gypaètes), la mésentente entre animaux forcés de coexister (il a fallu séparer des couples de gypaètes dont le mâle et la femelle ne se supportaient pas), etc. 
d'interventions, aussi balbutiant que la protection au début du siècle dernier. La chasse en fait partie. Pourquoi la chasse, pratique ancestrale s'il en est, relèverait-elle de l'éco-zoo-technie ? C'est que la chasse, dans les Alpes, a passablement changé. Le changement de terminologie en témoigne: les animaux ne sont plus tués, mais «prélevés » et une fraction croissante des chasseurs se définissent comme des «gestionnaires de la faune sauvage » (Dalla Bernadina, 1989). On pourrait voir là un simple procédé rhétorique, destiné à réhabiliter, en la « verdissant », une activité largement décriée. Mais les techniques et les pratiques des chasseurs ont elles aussi considérablement évolué. Les plans de chasse fixent un quota d'animaux à prélever et les chasseurs ne tirent plus n'importe quel animal, mais un individu de telle classe d'âge et éventuellement de tel sexe, qu'ils sont ensuite tenus de montrer à une commission de contrôle. À côté de la gestion cynégétique à laquelle ont dû se convertir les chasseurs montagnards, des captures contribuent à diminuer ponctuellement les densités de population. Chaque année, des marmottes sont soustraites à la zone centrale du parc national de la Vanoise pour être relâchées dans d'autres massifs ou simplement en dehors des prés de fauche où elles se sont établies. On voit poindre, depuis peu, de nouvelles méthodes de traitement des animaux sauvages, non plus physiques mais biologiques. Devant la difficulté à trouver de nouveaux preneurs de marmottes et les doutes concernant la capacité des captures à régler les problèmes des agriculteurs, une expérimentation de contraception a été entreprise en 2004 en zone centrale du parc national des Écrins. Les substances contraceptives qui ont été administrées aux rongeurs sont habituellement utilisées pour des animaux de compagnie comme les chiens et les chats. C'est l'amorce d'une extension à la faune sauvage de procédés jusqu'ici réservés à l'homme et aux animaux domestiques. L'expérimentation a provoqué un certain nombre de réactions et de critiques, notamment dans les milieux de la protection de la nature, habitués à protéger et à sauvegarder la vie animale et non à la réduire, et elle n’a pas été reconduite en $2005^{10}$. Mais, quel que soit son avenir, il semble que l'on soit décidément entré dans l’ère de la production d'animaux « sauvagement artificiels » (Larrère, 1994).

\section{Les réactions contrastées des sociétés alpines}

Le développement et l'extension de la grande faune sauvage ont réjoui plusieurs catégories d'acteurs. Parmi eux, bien entendu, les naturalistes, les protecteurs et les gestionnaires de la nature, qui se sont battus, souvent avec acharnement, pour réintroduire des espèces qui avaient disparu, comme le gypaète, ou pour sauvegarder celles qui allaient connaître le même sort, comme le bouquetin. Les effectifs européens de ce dernier étaient estimés à moins de cent en 1820 ; ils dépassaient les vingt-cinq mille en 1990 (Gauthier et Villaret, 1990 : 97). Pour tous ceux qui ont participé à ce « grand retour » ${ }^{11}$, c'est une réussite, et une victoire.

\section{Une économie naissante autour des animaux sauvages}

Les positions des chasseurs sont plus mitigées. Une partie d'entre eux s'inquiètent de la présence de grands prédateurs et de son impact sur les populations d'ongulés sauvages. Quant aux plus anciens, ils sont nombreux à estimer que la chasse au chamois, qui reste bien souvent le gibier roi, est devenue trop facile et trop surveillée, depuis l'instauration des plans de chasse : «Moi j’ai abandonné à ce moment-là. J'ai pas fait une seule saison avec le plan de chasse. J'ai pas essayé, j'ai vu comment ça allait se passer. Toutes ces choses imposées, on peut

10 Pour une présentation détaillée et une analyse de cette expérimentation de contraception, voir (Mauz et Granjou, 2006).

11 « Le grand retour » est le titre d'un film consacré à l'histoire du bouquetin, coproduit par le parc national de la Vanoise et Anne et Erik Lapied, 1989, 30’. 
pas aller où on veut, on peut pas y aller le jour où on veut, on peut pas... » (un ancien chasseur savoyard). Mais beaucoup se réjouissent de pouvoir " prélever », à côté des chamois, des cerfs, des chevreuils, des sangliers ou des mouflons. Quelques-uns commencent à dire que «le loup est un magnifique animal de chasse $»^{12}$. Le chasseur montagnard est passé de la pénurie à l'abondance et il a désormais l'embarras du choix ; l'évolution des tableaux de chasse est à cet égard éloquente ${ }^{13}$. La chasse est en outre susceptible de rapporter des revenus substantiels aux collectivités montagnardes et aux services forestiers. Certaines communes ont franchi le pas et louent tout ou partie de leur territoire de chasse à des amateurs fortunés. En France, l'Office national des forêts propose des journées de chasse et des stages de formation à la chasse au grand gibier dans des forêts domaniales et dans la réserve nationale de chasse et de faune sauvage des Bauges : on peut acheter, plutôt chèrement ${ }^{14}$, le droit de tuer un cerf, un chamois, un mouflon, un chevreuil ou un sanglier en étant guidé par un agent. Les amateurs sont hébergés dans des hôtels, auberges et gîtes situés à proximité des territoires de chasse : on assiste ainsi à la création d’une économie, et de métiers, autour des animaux sauvages.

Si la grande faune sauvage est devenue une nouvelle ressource à exploiter, ce n'est pas seulement parce qu'elle se chasse. Comme on l'a déjà suggéré, une grande partie du public apprécie tout simplement de voir des animaux. Les professionnels du tourisme ont vite saisi l'intérêt de cet engouement : des safaris photos sont organisés et de nombreuses sorties en montagne encadrées par des professionnels ont pour but affiché l'observation et l'approche des grands ongulés. Dans le Mercantour et dans le Vercors, des agences proposent à leurs clients de partir « sur les traces du loup ». Une nouvelle forme de tourisme émerge, que l'on peut qualifier d'animalier ou, si l'on préfère, de zoologique.

L'importance qu'ont prise les animaux sauvages se manifeste jusque dans les villages et les stations. Peints ou sculptés sur les façades des maisons, statufiés au beau milieu de la place publique, ils sont désormais partout et paraissent largement adoptés, bien au-delà du cercle des initiés.

\section{Le désarroi des éleveurs et des agriculteurs}

Cependant, la multiplication et la venue d'animaux sauvages ont aussi mécontenté une partie des habitants. Elles se sont produites et continuent de se produire dans des mondes déjà peuplés d'animaux domestiques, d'éleveurs et d'agriculteurs, où elles génèrent des problèmes de plusieurs ordres. Les pathologies constituent une première source d'inquiétudes et de récriminations. Elles se transmettent d'autant plus facilement entre animaux domestiques et sauvages appartenant à des espèces proches, voire à la même espèce (comme le mouflon et le mouton, le bouquetin et la chèvre, le sanglier et le cochon), qu'ils se côtoient de plus en plus souvent et de plus en plus longtemps. Dans le Beaufortain, l'identification de foyers de brucellose chez le chamois au cours des années 1990 avait fortement inquiété les éleveurs et les vétérinaires et l'extinction de ces foyers a été accueillie avec soulagement. De manière générale, les éleveurs craignent que les animaux sauvages ne constituent un

12 Propos tenus par le président de la fédération des chasseurs de la Savoie le 31 mai 2005, lors du débat “ Vivre avec le loup ”, organisé à Chambéry par France Bleu Pays de Savoie.

13 Les tableaux de chasse peuvent être consultés sur http://www.oncfs.gouv.fr/events/point_faune/mammifere.php

14 À titre d'exemple, une journée de chasse à l'approche guidée dans la forêt domaniale de Lente, à Saint-Jeanen-Royans (Drome) coûte $280 €$ (200€ du 15 novembre à la fermeture générale). S'ajoute le prix du tir, très variable en fonction de l'animal (de $55 €$ pour une chevrette à plus de $1200 €$ pour un cerf adulte). L'offre cynégétique de l'Onf, consultable sur http://www.onf.fr/foret/dossier/chasse/index.htm, est maintenant très diversifiée. 
réservoir de maladies qu'eux-mêmes s'efforcent de combattre et d'éliminer dans les troupeaux domestiques, parfois par des moyens radicaux comme l'abattage. Autre sujet de préoccupations et de conflits : la concurrence alimentaire entre ongulés domestiques et sauvages, ces derniers étant localement accusés de prélever une part substantielle de la ressource fourragère, notamment au printemps. Les agents du parc national de la Vanoise demandent régulièrement à des stagiaires de repousser les hardes de bouquetins qui descendent au mois de mai dans des prairies de fauche et des champs de luzerne. Dernier écueil, et non le moindre : les dégâts causés par les animaux sauvages, parmi lesquels on peut distinguer les dommages aux terrains, quelquefois très sérieux (prairies de fauche et pelouses d'altitude «labourées » par des sangliers), et les attaques de troupeaux domestiques par de grands prédateurs. Ce sont ces dégâts qui suscitent actuellement les tensions les plus vives : ils sont en effet coûteux et, même si des progrès significatifs ont été accomplis dans la protection des troupeaux ovins contre les attaques de loups ${ }^{15}$, ils restent difficiles à prévenir et impossibles à éliminer totalement. D'où une opposition de la grande majorité des agriculteurs et des éleveurs, susceptible d'aller jusqu'à des conflits avec les chasseurs, les protecteurs ou les gestionnaires, et jusqu’à une résistance, active ou passive, légale ou illégale : des manifestations sont organisées contre les loups ; des sangliers et de grands prédateurs sont braconnés dans plusieurs pays de l'arc alpin. Cette résistance et ces conflits sont très variables selon les pays, voire les régions ${ }^{16}$. Mais partout, le développement de la grande faune sauvage et, à nouveau, pas seulement des grands prédateurs, inquiète et ébranle le pastoralisme, jusque dans ses fondements.

Longtemps, les Alpes, leurs habitants et les troupeaux domestiques ont en effet formé un tout indissociable. Dans L'Homme et la montagne (1934), le géographe Jules Blache, disciple de Raoul Blanchard, définissait les montagnes en général et les Alpes en particulier comme le domaine des migrations pastorales (Sgard, 2004). Parallèlement, les sociétés alpines ont été qualifiées de «sociétés de la vache » (Poche, 1999) ou, dans une moindre mesure, du mouton ou de la chèvre, en tout cas de l'ongulé domestique. Dans ces sociétés alpines passées maîtresses dans l'exploitation agropastorale des ressources naturelles, la chasse ne jouait qu'un rôle tout à fait mineur ${ }^{17}$ et les animaux sauvages ont été condamnés à demeurer rares et farouches (Dalla Bernadina, 1988). Dès qu'ils en ont eu les moyens, les montagnards ont éliminé les créatures les plus gênantes pour l'agriculture et l'élevage, à commencer par les grands prédateurs. Ils ont relégué les autres aux confins de l'espace communal, généralement sans grand souci de préservation : «Ils tiraient le chamois qu'ils voyaient, c'était tout. Y avait pas ni sexe ou pas sexe. Bon, une chèvre suitée $e^{18}$, ils la tiraient peut-être pas, c'était tout. Mais le reste, tout ce qui était pas suité, ils s'occupaient pas de savoir ce que c'était » (un chasseur de chamois savoyard). Ces mondes alpins avaient cependant leurs spécialistes des rapports au sauvage (Mauz, 2005 : 145155) : dans chaque commune, un très petit nombre d’hommes, et parfois même un seul, fréquentaient assidûment

15 Le séminaire «Loup élevage, s’ouvrir à la complexité », qui s’est tenu à Aix-en-Provence les 15 et 16 juin 2006, a permis de faire le point sur les différentes mesures (regroupement nocturne des troupeaux, introduction de chiens de protection, renforcement du gardiennage et embauche d'aides-bergers), et de mettre en évidence les acquis et les questions en suspens, quinze ans après le retour des loups dans les Alpes françaises.

16 Ce n'est probablement pas un hasard si les oppositions à la venue de grands prédateurs sont particulièrement vives dans plusieurs cantons suisses, qui s'affirment comme le foyer de la civilisation pastorale alpestre.

17 Certaines espèces ont cependant joué un rôle non négligeable dans l'économie locale. C'est le cas des marmottes, chassées pour leur peau, dont la vente, dit-on, pouvait rapporter aux bergers autant que la garde des troupeaux, et pour leur viande: "la marmotte, c'était la viande des bergers » (un agriculteur retraité). Relativement faciles à maintenir en dehors des terres cultivées et à capturer, les marmottes, qui de surcroît dorment une bonne partie de l'année et peuvent s'apprivoiser, ont longtemps fait partie de ce sauvage exploitable que les montagnards n'ont pas cherché à éliminer.

18 Mère accompagnée d'un petit de l'année. 
la montagne, s’adonnaient à la chasse avec une passion et une intensité hors du commun, et servaient de médiateurs au sauvage. C'est à ces individus, un peu marginaux et souvent affligés d'une tare physique ou morale (Dalla Bernardina, 2004), que l’on s’adressait au village pour toute question relative au sauvage.

La population agricole n’a cessé de décliner tout au long du vingtième siècle et l'élevage alpin, en dépit de brillantes réussites, souffre de nombreux maux. Les éleveurs savent bien que la faune sauvage n'est que l'un d'entre eux. Mais celui-là est bien visible, lui, et il témoigne de l'existence d'un projet d'ensauvagement des Alpes qui semble aller à l'encontre de celui qui a été poursuivi pendant des siècles.

\section{Entre tout sauvage et tout domestique, la possibilité d'une autre voie ?}

Les protecteurs et les gestionnaires des espaces protégés soulignent que plusieurs espèces demeurent extrêmement fragiles et menacées, et que leur pérennité est loin d’être acquise. L’essor de la grande faune sauvage a certes été spectaculaire mais son statut, tant juridique que biologique, reste des plus précaires. Il suffirait d'une épidémie de gale sarcoptique, affirment ainsi les spécialistes du bouquetin, pour laminer les populations actuelles et réduire à néant les résultats obtenus au cours des dernières décennies. On sait combien la présence des grands prédateurs est contestée ; en ce qui les concerne, la situation n’est clairement pas stabilisée. Aussi les naturalistes, les protecteurs et les gestionnaires estiment-ils que l’on pourrait très bien « revenir à la case départ » et se retrouver, d’ici peu, avec une faune sauvage aussi mal en point qu'il y a peu. Il conviendrait donc de ne pas relâcher la vigilance et de poursuivre les efforts en faveur de la réhabilitation de la grande faune.

Ce que craignent pour leur part les éleveurs, c’est l'écroulement, à terme, du monde pastoral et le remplacement d’une montagne domestiquée par une montagne ensauvagée. Les propos d’un éleveur des Alpes françaises du Nord sont, à cet égard, assez significatifs : «nos jeunes, ici, ne pourront plus faire du foin, alors ça va être vite $v u$, il faudra bien qu'ils aillent le faire ailleurs, le foin [Q : pourquoi ils pourront plus faire du foin ?] Ce sera tout massacré par les cochons ! Mangé par les sangliers, et puis les chevreuils, les cerfs et compagnie, alors !». Si la prédiction de cet éleveur devait se réaliser, si donc l'animal domestique et le pastoralisme devenaient aussi marginaux que l'ont si longtemps été l'animal sauvage et la chasse, c'est toute une culture, tout un rapport au monde qui disparaîtraient avec eux.

Une troisième voie paraît possible entre ces deux scénarios extrêmes. Elle consiste en une coexistence des faunes domestique et sauvage et des hommes qui leur sont attachés. Ce n'est certainement pas la plus facile. Le plus facile, en effet, serait que les Alpes n'abritent que des animaux sauvages, ou que des animaux domestiques. On éviterait ainsi que des loups ne s’attaquent aux brebis, que des chamois ne contractent la brucellose en côtoyant des troupeaux non indemnes, et que des gens qui ne partagent pas les mêmes points de vue et ont des expériences différentes des animaux ne s'affrontent périodiquement. D’aucuns en rêvent, ou rêvent du moins d'une franche domination, tel ce responsable d'une grande association de protection de la nature s'exprimant sur la place des agriculteurs dans les aires protégées : « un parc national n'est pas une zone d'activités économiques. On devrait dire aux éleveurs : "On veut bien, par gentillesse, vous autoriser à y mettre les pieds pour essayer de tirer quelque chose, on ferme les yeux. Mais alors, on ne vous entend pas, on ne vous voit pas, vous passez inaperçus, vous êtes transparents. Si vous nous posez des problèmes...” [...] Il faut qu'ils sachent, avant d'y aller, qu'il y a un risque, qu'ils vont avoir des soucis mais qu'ils doivent comprendre leur situation et rester discrets. C'est pour ça qu'il faut leur faire signer des contrats. À ce moment-là, nous, on acceptera qu'il y ait quelques activités 
possibles d'agriculture. » On peut recueillir chez leurs éleveurs et leurs alliés des propos tout à fait symétriques. Face à ces positions tranchées, il importe de rappeler que la diversité et la facilité font rarement bon ménage. Si l'on veut de la diversité, ce que tous affirment régulièrement, il faut accepter l'idée qu'il y aura des difficultés, qu'il y aura des conflits et que les conflits ne sont pas la pire chose qui nous menace.

\section{Conclusion : les conditions de la coexistence}

Que faut-il pour s'engager dans cette voie et échapper aux deux autres ? Il faut des moyens. Moyens humains, notamment sur les alpages, où, en bien des endroits, la main-d'œuvre est insuffisante pour permettre la cohabitation des animaux sauvages et domestiques. S'il faut des bergers pour seconder les éleveurs, il faut aussi des techniciens, et des chercheurs, pour mieux connaître et mieux comprendre les relations entre les espèces animales et entre les hommes et les animaux. Moyens techniques, pour que les difficultés et les conflits n’atteignent pas un niveau insupportable. Des progrès et des innovations ont déjà été réalisés dans ce domaine mais il reste à l'évidence un long chemin à parcourir pour que les faunes domestique et sauvage parviennent à coexister dans les Alpes non pas sereinement, mais sans que l'une finisse par l'emporter sur l'autre. Une génération ne suffira peut-être même pas à inventer, à forger et à maîtriser tous les outils, toutes les parades et tout le vocabulaire qui actuellement font défaut. Moyens financiers, parce que le recrutement et le logement de bergers et d'aides-bergers, la mise au point et l'emploi de mesures de protection ont un coût et parce que ces mesures, si efficaces soient-elles, n'élimineront pas tous les problèmes et ne permettront pas d'éviter complètement le recours aux indemnisations.

Il faut encore se méfier des dogmatismes de tout poil, et ne considérer aucune situation comme simple, acquise et définitive. Une espèce abondante peut rapidement être menacée et réciproquement. Si des innovations techniques contribuent incontestablement à résoudre ou à améliorer des situations qui paraissaient critiques, elles sont rarement partout applicables et les contextes locaux doivent être pris en considération, dans toutes leurs dimensions écologiques, économiques et sociales. Les animaux, dont on tend à sous-estimer l'intelligence et la capacité d'adaptation, finissent en outre souvent par trouver des failles dans les dispositifs mis en place. Dans ces conditions, les solutions techniques que l'on adopte, les statuts juridiques que l'on assigne et les accords que l'on passe ne sauraient être intangibles. Ils doivent être ajustés aux singularités du moment et du lieu et très régulièrement réexaminés et renégociés, avec l'ensemble des acteurs impliqués, si difficile que cela soit parfois. Ce dont nous avons besoin, en définitive, c'est d'une politique de la faune, sauvage et domestique. Une politique au sens noble, celui que lui a notamment donné Hannah Arendt : une politique fondée sur la pluralité, la diversité et les limitations réciproques.

\section{Bibliographie}

Abry, C., Bessat, H., Carpitelli, E., 1998. Les racines de l'alpe. L’Alpe, 1, 6 p.

Arendt, H. (1986) : Vies politiques. Paris, Gallimard.

Baratay, E. et Hardouin-Fugier E., 1998. Zoos. Histoire des jardins zoologiques en Occident (XVI ${ }^{e}$-XX ${ }^{e}$ siècle).

Paris, La Découverte.

Blache, J., 1934. L'Homme et la montagne. Paris, Gallimard.

Dalla Bernadina, S., 1989. L'invention du chasseur écologiste : un exemple italien. Terrain 13 : 130-139.

Debarbieux, B., 1995. Tourisme et montagne. Paris, Economica. 
De Planhol, X., 2004. Le paysage animal. L’homme et la grande faune : une zoogéographie historique. Paris, Fayard.

Fischer, F., 2000. Citizens, experts and the environment. The politics of local knowledge. Durham and London, Duke university

press.

Gauthier, D., Villaret J.-C., 1990 : La réintroduction en France du bouquetin des Alpes, Revue d'écologie la terre et la vie), actes publiés par la Société nationale de protection de la nature, Suppl. 5: 97-120. Hainard, R., 1997. Mammifères sauvages d'Europe. Paris, Lausanne, Delachaux et Niestlé. Larrère, R., 1994. Sauvagement artificiel. Le courrier de l'environnement de l'Inra, 21: 35-37. Lecomte, J., 1989. Introduire, réintroduire, voilà la question. Le courrier de la cellule environnement, 6 : 21-30.

Loire, C., 1984. Le loup à la fin du XXème siècle. Thèse de l'Ecole nationale vétérinaire de Lyon. 226 p. Luxereau, A., 2004. Des animaux ni sauvages ni domestiques, les "girafes des Blancs" au Niger. Anthropozoologica, 39

289-300.

Madden, F., 2004. Social science perspectives on the prevention and mitigation of human-mountain gorilla conflict in southwest Uganda, 10th international symposium on society and resource management, June 2-6, Keystone,

Colorado,

USA.

Massol, G., 2005. Le mouflon. Quel avenir pour les grands mammifères sauvages de montagne face à des activités humaines croissantes ? Chez l'auteur. Mauz, I., 2003. Histoire et mémoires du parc national de la Vanoise. 1921-1971 : la construction. Revue de géographie alpine, collection Ascendances. Mauz, I., 2005. Gens, cornes et crocs. Paris, Cemagref, Cirad, Ifremer, Inra.

Mauz, I., Granjou, C., 2006. L'« affaire » des marmottes de Prapic. Des frontières familières à l'épreuve d'une expérimentation de contraception animale, Colloque « Homme et animal, la question des frontières », Dijon, 1617 mai 2006.

Micoud, A., 1993. Vers un nouvel animal sauvage : le sauvage “ naturalisé vivant” ? Natures-Sciences-Sociétés, 1

Poche, B., 1999. Le monde bessanais. Société et représentation. Paris, éditions du Cnrs

Callon, M., Lascoumes, P. et al., 2001. Agir dans un monde incertain. Essai sur la démocratie technique. Paris, Seuil.

Dalla Bernadina, S., 1988. Hédonistes et ascètes. “ Latins ” et “ septentrionaux ” à la chasse au chamois dans les Alpes italiennes. Le Monde alpin et rhodanien (La haute montagne. Vision et représentations) : 165-185.

Dalla Bernadina, S., 1989. L'invention du chasseur écologiste : un exemple italien. Terrain 13 : 130-139.

Debarbieux, $\quad$ B., $1995 . \quad$ Tourisme et montagne. Paris, Economica. De Planhol, X., 2004. Le paysage animal. L’homme et la grande faune : une zoogéographie historique. Paris, Fayard.

Fischer, F., 2000. Citizens, experts and the environment. The politics of local knowledge. Durham and London, Duke university

press.

Gauthier, D., Villaret J.-C., 1990 : La réintroduction en France du bouquetin des Alpes, Revue d'écologie la terre et la vie), actes publiés par la Société nationale de protection de la nature, Suppl. 5: 97-120. Hainard, R., 1997. Mammifères sauvages d'Europe. Paris, Lausanne, Delachaux et Niestlé. 
Larrère, R., 1994. Sauvagement artificiel. Le courrier de l'environnement de l’Inra, 21: 35-37. Lecomte, J., 1989. Introduire, réintroduire, voilà la question. Le courrier de la cellule environnement, 6 : 21-30.

Loire, C., 1984. Le loup à la fin du XXème siècle. Thèse de l'Ecole nationale vétérinaire de Lyon. 226 p. Luxereau, A., 2004. Des animaux ni sauvages ni domestiques, les "girafes des Blancs" au Niger. Anthropozoologica,

Madden, F., 2004. Social science perspectives on the prevention and mitigation of human-mountain gorilla conflict in southwest Uganda, 10th international symposium on society and resource management, June 2-6, Keystone, Colorado,

USA. Massol, G., 2005. Le mouflon. Quel avenir pour les grands mammifères sauvages de montagne face à des activités humaines croissantes ? Chez l'auteur. Mauz, I., 2003. Histoire et mémoires du parc national de la Vanoise. 1921-1971 : la construction. Revue de géographie alpine, collection Ascendances. Mauz, I., 2005. Gens, cornes et crocs. Paris, Cemagref, Cirad, Ifremer, Inra.

Mauz, I., Granjou, C., 2006. L'« affaire » des marmottes de Prapic. Des frontières familières à l'épreuve d'une expérimentation de contraception animale, Colloque « Homme et animal, la question des frontières », Dijon, 1617 mai 2006.

Micoud, A., 1993. Vers un nouvel animal sauvage : le sauvage “ naturalisé vivant” ? Natures-Sciences-Sociétés, 1

Poche, B., 1999. Le monde bessanais. Société et représentation. Paris, éditions du Cnrs Kleitz, G., 2003. Ruling by nature. Analysing the implementation of nature conservation projects in rural areas. A case study in Northern Zimbabwe. Degree of philosophy in development studies, University of Sussex: 242 p. Dalla Bernadina, S., 1989. L'invention du chasseur écologiste : un exemple italien. Terrain 13 : 130-139.

Debarbieux, $\quad$ B., $1995 . \quad$ Tourisme et montagne. Paris, Economica. De Planhol, X., 2004. Le paysage animal. L’homme et la grande faune : une zoogéographie historique. Paris, Fayard.

Fischer, F., 2000. Citizens, experts and the environment. The politics of local knowledge. Durham and London, Duke university

press.

Gauthier, D., Villaret J.-C., 1990 : La réintroduction en France du bouquetin des Alpes, Revue d'écologie la terre et la vie), actes publiés par la Société nationale de protection de la nature, Suppl. 5: 97-120. Hainard, R., 1997. Mammifères sauvages d'Europe. Paris, Lausanne, Delachaux et Niestlé. Larrère, R., 1994. Sauvagement artificiel. Le courrier de l'environnement de l'Inra, 21: 35-37. Lecomte, J., 1989. Introduire, réintroduire, voilà la question. Le courrier de la cellule environnement, 6 : 21-30.

Loire, C., 1984. Le loup à la fin du XXème siècle. Thèse de l'Ecole nationale vétérinaire de Lyon. 226 p. Luxereau, A., 2004. Des animaux ni sauvages ni domestiques, les "girafes des Blancs" au Niger. Anthropozoologica,

Madden, F., 2004. Social science perspectives on the prevention and mitigation of human-mountain gorilla conflict in southwest Uganda, 10th international symposium on society and resource management, June 2-6, Keystone, Colorado,

USA.

Massol, G., 2005. Le mouflon. Quel avenir pour les grands mammifères sauvages de montagne face à des 
activités

humaines

croissantes?

Chez

l'auteur.

Mauz, I., 2003. Histoire et mémoires du parc national de la Vanoise. 1921-1971 : la construction. Revue de géographie

alpine,

collection

Ascendances.

Mauz, I., 2005. Gens, cornes et crocs. Paris, Cemagref, Cirad, Ifremer, Inra.

Mauz, I., Granjou, C., 2006. L'« affaire » des marmottes de Prapic. Des frontières familières à l'épreuve d'une expérimentation de contraception animale, Colloque « Homme et animal, la question des frontières », Dijon, 1617 mai

2006.

Micoud, A., 1993. Vers un nouvel animal sauvage: le sauvage " naturalisé vivant” ? Natures-SciencesSociétés, 1

202-210.

Poche, B., 1999. Le monde bessanais. Société et représentation. Paris, éditions du Cnrs Kleitz, G., 2003. Ruling by nature. Analysing the implementation of nature conservation projects in rural areas. A case study in Northern Zimbabwe. Degree of philosophy in development studies, University of Sussex: 242 p. Dalla Bernadina, S., 1989. L'invention du chasseur écologiste : un exemple italien. Terrain 13 : 130-139.

Debarbieux, $\quad$ B., $1995 . \quad$ Tourisme et montagne. Paris, Economica.

De Planhol, X., 2004. Le paysage animal. L’homme et la grande faune : une zoogéographie historique. Paris, Fayard.

Fischer, F., 2000. Citizens, experts and the environment. The politics of local knowledge. Durham and London, Duke university

press.

Gauthier, D., Villaret J.-C., 1990 : La réintroduction en France du bouquetin des Alpes, Revue d'écologie la terre et la vie), actes publiés par la Société nationale de protection de la nature, Suppl. 5 : 97-120. Hainard, R., 1997. Mammifères sauvages d'Europe. Paris, Lausanne, Delachaux et Niestlé. Larrère, R., 1994. Sauvagement artificiel. Le courrier de l'environnement de l'Inra, 21: 35-37. Lecomte, J., 1989. Introduire, réintroduire, voilà la question. Le courrier de la cellule environnement, 6 : 21-30.

Loire, C., 1984. Le loup à la fin du XXème siècle. Thèse de l'Ecole nationale vétérinaire de Lyon. 226 p. Luxereau, A., 2004. Des animaux ni sauvages ni domestiques, les "girafes des Blancs" au Niger. Anthropozoologica,

Madden, F., 2004. Social science perspectives on the prevention and mitigation of human-mountain gorilla conflict in southwest Uganda, 10th international symposium on society and resource management, June 2-6, Keystone,

Colorado,

USA.

Massol, G., 2005. Le mouflon. Quel avenir pour les grands mammifères sauvages de montagne face à des activités humaines croissantes?

Chez

l'auteur.

Mauz, I., 2003. Histoire et mémoires du parc national de la Vanoise. 1921-1971 : la construction. Revue de géographie alpine, collection

Ascendances.

Mauz, I., 2005. Gens, cornes et crocs. Paris, Cemagref, Cirad, Ifremer, Inra.

Mauz, I., Granjou, C., 2006. L'« affaire » des marmottes de Prapic. Des frontières familières à l'épreuve d'une expérimentation de contraception animale, Colloque "Homme et animal, la question des frontières », Dijon, 16-17 mai 2006. 
Micoud, A., 1993. Vers un nouvel animal sauvage: le sauvage "naturalisé vivant" ? Natures-Sciences$\begin{array}{lll}\text { Sociétés, } & 1 & \text { (3) : 202-210. }\end{array}$

Poche, B., 1999. Le monde bessanais. Société et représentation. Paris, éditions du Cnrs

Sgard, A., 2004. Un moment de la construction du savoir sur la montagne : Jules Blache dans L'Homme et la Montagne (1934), in L'effet géographique. Construction sociale, appréhension cognitive et configuration matérielle des objets géographiques, sous la direction de Bernard Debarbieux et Marie-Christine Fourny, pp. 3754.

Stephen, L., 2003. Le terrain de jeu de l'Europe. Paris, Hoëbeke.

Vourc'h, A., Pelosse, V. 1992. Le lynx a-t-il le mauvais œil ? La terre outragée. Les experts sont formels !, J. Theys, B. Kalaora. Paris, Éditions Autrement, p. 126-129. 